University of Nebraska - Lincoln

DigitalCommons@University of Nebraska - Lincoln

2008

Synthesis and Characterization of an ABC Miktoarm Star Terpolymer of Cyclohexadiene, Styrene, and 2-Vinylpyridine

David Uhrig

Kunlun Hong

Jimmy W. Mays

S. Michael Kilbey II

Phillip F. Britt

Follow this and additional works at: https://digitalcommons.unl.edu/usdoepub

Part of the Bioresource and Agricultural Engineering Commons

Uhrig, David; Hong, Kunlun; Mays, Jimmy W.; Kilbey II, S. Michael; and Britt, Phillip F., "Synthesis and Characterization of an ABC Miktoarm Star Terpolymer of Cyclohexadiene, Styrene, and 2-Vinylpyridine" (2008). US Department of Energy Publications. 72.

https://digitalcommons.unl.edu/usdoepub/72

This Article is brought to you for free and open access by the U.S. Department of Energy at DigitalCommons@University of Nebraska - Lincoln. It has been accepted for inclusion in US Department of Energy Publications by an authorized administrator of DigitalCommons@University of Nebraska - Lincoln. 


\section{Synthesis and Characterization of an $\mathrm{ABC}$ Miktoarm Star Terpolymer of Cyclohexadiene, Styrene, and 2-Vinylpyridine}

\section{David Uhrig,* Kunlun Hong, Jimmy W. Mays, S. Michael Kilbey II, and Phillip F. Britt}

The Center for Nanophase Materials Sciences, Oak Ridge
National Laboratory, Oak Ridge, Tennessee 37831

Received August 13, 2008

Revised Manuscript Received October 15, 2008

Several research groups have expended great effort toward the preparation of miktoarm (mixed-arm) star copolymers and terpolymers. ${ }^{1-9}$ We describe herein a novel synthesis, using anionic polymerization, of a poly (cyclohexadiene) [PCHD]-polystyrene [PS]-poly(2-vinylpyridine) [P2VP] miktoarm star terpolymer.

Previously, miktoarm $\mathrm{A}_{2} \mathrm{~B}$ and $\mathrm{A}_{3} \mathrm{~B}$ star polydiene copolymers comprised partially of poly(cyclohexadiene) have been synthesized by Tsoukatos and Hadjichristidis by employing chlorosilane linking techniques. ${ }^{10}$ More recently, an ABC star terpolymer of PS, polyisoprene, and PCHD was prepared by Yang and co-workers by using meta-double-diphenylethylenebased linking chemistry. ${ }^{11}$

The three arms of the novel $\mathrm{ABC}$ mixed-arm star terpolymer described herein were chosen purposefully to create, upon exposure to a silica or mica surface, a conformationally asymmetric binary brush having a P2VP "anchor" block with both PS and high 1,4-PCHD "buoys". The chemical dissimilarity of the arms and the short-term longevity of poly(cyclohexadienyl) macroanions combine to pose a difficult synthetic challenge. For example, the cyclohexadiene block needs to be synthesized in nonpolar media without the presence of P2VP as polar components complicate both control of the PCHD anion stability and the resultant microstructure of the PCHD polymer obtained. In contrast, polymerization of 2-vinylpyridine is most successfully achieved in tetrahydrofuran (THF) at low temperatures, so switching of polymerization media during the synthesis was required.

The target polymer was sought through the use of a 1,1-diphenylethylene (DPE)-based strategy as shown in Scheme 1. Employing anionic polymerization techniques previously detailed, ${ }^{12-16}$ PCHDLi was first prepared from the polymerization of cyclohexadiene using the initiator system of sec-BuLi/ DABCO; this PCHDLi was added to bis(1-phenylethenyl)benzene (PEB), and the product was quenched with methanol. The product was precipitated many times to remove excess PEB (iteratively into hexane, methanol, and hexane, dissolving each time in anhydrous benzene) and thereafter prepared as a concentrated benzene solution and delivered to a glass apparatus. In this apparatus, the PCHD-PEB was stripped of its solvent under vacuum and redissolved with fresh anhydrous benzene three times, after which the PCHD-PEB was left for 3 days under high vacuum; the dried PCHD-PEB was finally dissolved into anhydrous benzene and distributed into ampules. Separately, PSLi was prepared from styrene and sec-BuLi in benzene and collected to a large cylindrical ampule.

The first attempt to add PCHDLi to PEB was made by using the 1,3-PEB isomer. While in the case of Yang and co-workers ${ }^{11}$ this strategy did work well, in our case it did not work in the

\footnotetext{
* To whom correspondence should be addressed.
}

desired manner. There was a $\sim 30 \%$ amount of dimer in the product, indicating the addition of two arms of PCHD to the PEB instead of only one as desired, even though PEB was used in a 3-fold amount relative to the PCHDLi (see Figure 1A). One way to limit the amount of dimerization is to employ a large amount of polar solvent (THF) in the end-capping reaction, which is the method of Yang. ${ }^{11,17}$ We were reluctant to depart from benzene here because, in our case, the macroanion involved was PCHDLi-DABCO, which can be a more sensitive/ troublesome intermediate than other macroanions. Another option is alteration of the PEB structure and reactivity. Following the ideas and work of Paraskeva and Hadjichristidis, ${ }^{18}$ the 1,4PEB isomer was employed with greater success (see Figure 1B). Briefly, the addition of a first carbanion to one double bond of 1,4-PEB more effectively repels a second carbanion from opening the neighboring double bond (through mesomeric delocalization of charge), even in nonpolar media, until the first anion is quenched.

A second failed attempt involved adding PSLi to the PCHD-1,4-PEB adduct, which was largely attended by quenching a majority of the PSLi macroanion (see Figure 1C). It seems possible to us that the problem here is symptomatic of the shortterm stability of PCHDLi in the presence of DABCO and its ability to chemically attack the additive upon starvation of monomer. Comparison of the RI and UV detector responses in the size exclusion chromatography (SEC) eluogram of PCHD-PEB (see Figure 1D) seems to indicate that not all of the PCHD was end-capped with 1,4-PEB but, rather, that a significant quantity had deactivated before it could react with PEB, as indicated by a low molecular weight tail which exists only in the RI trace. For precedence to this explanation, see the work by Poshyachinda, Edwards, and Johnson, ${ }^{19}$ in which the PBLi attack of 1,2-bis(piperidino)ethane after long reaction times was observed and characterized by SEC and IR measurements. If this type of side reaction was also our problem, then small amounts of macromolecular secondary amine would of course be able to deactivate the incoming PSLi before it could react with the PEB functionality.

The third attempt proceeded successfully by using a colorimetric organolithium titration of the unremovable protic species, which allowed subsequent addition of PSLi to the PCHD-PEB adduct to progress more cleanly. This star terpolymer was prepared by using the apparatus shown in Figure 2. The apparatus was evacuated on the high-vacuum line and detached. A solution of $2.23 \mathrm{~g}$ of PCHD-PEB (estimated, $163 \mu \mathrm{mol}$ ) in $40 \mathrm{~mL}$ of benzene was introduced to the reactor from an ampule appended above, and the ampule was removed. A titrating solution containing purified benzene, $\sim 185 \mu \mathrm{mol}$ of $n$-BuLi, 0.15 equiv of DPE (for colorimetric end point determination), and 10 equiv of THF (to ensure no free DPE lingered and to expedite the titration procedure) was prepared in the left cylinder. The titrating solution was added slowly to the reactor until a steadfast golden hue remained-this end point occurred after the addition of $\sim 93 \mu \mathrm{mol}$ of organolithium. The side appendage used for the titration was then detached. Next, $3.80 \mathrm{~g}$ of PSLi in benzene (estimated, $\sim 140 \mu \mathrm{mol}$ ) was added to the PCHD-PEB adduct, and the resultant mixture was concentrated to a volume of $\sim 50 \mathrm{~mL}$, by gently back-distilling benzene from the reactor to the PSLi cylinder. The PSLi cylinder was detached. This concentrated mixed solution became quite orange-red and was allowed to age 1 day at room temperature. 
Scheme 1

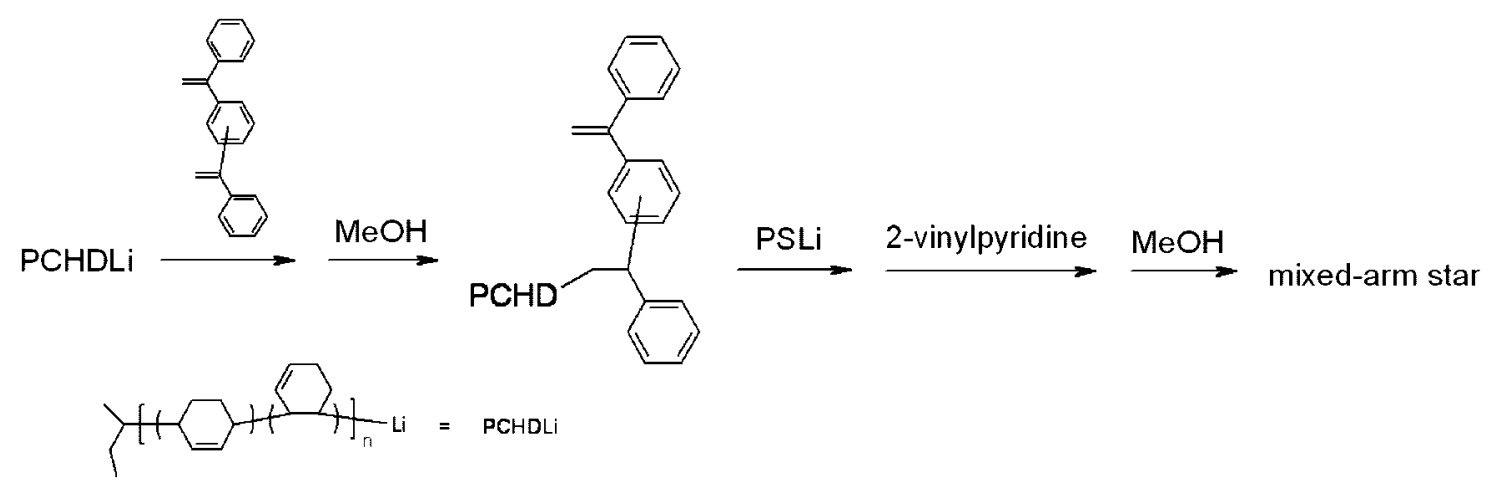

The apparatus was reaffixed to the high-vacuum line by using the latent break-seal entry. This new glass construction is not shown but was crafted to permit the sequential in-distillation of THF (on the vacuum line), followed by 2 -vinylpyridine (in a nearby affixed ampule) and, finally, methanol. During the first step, THF was in-distilled to the reactor as the temperature was simultaneously lowered to $-78^{\circ} \mathrm{C}$, resulting in a solution of the polymeric adduct in majority THF minority benzene (THF/
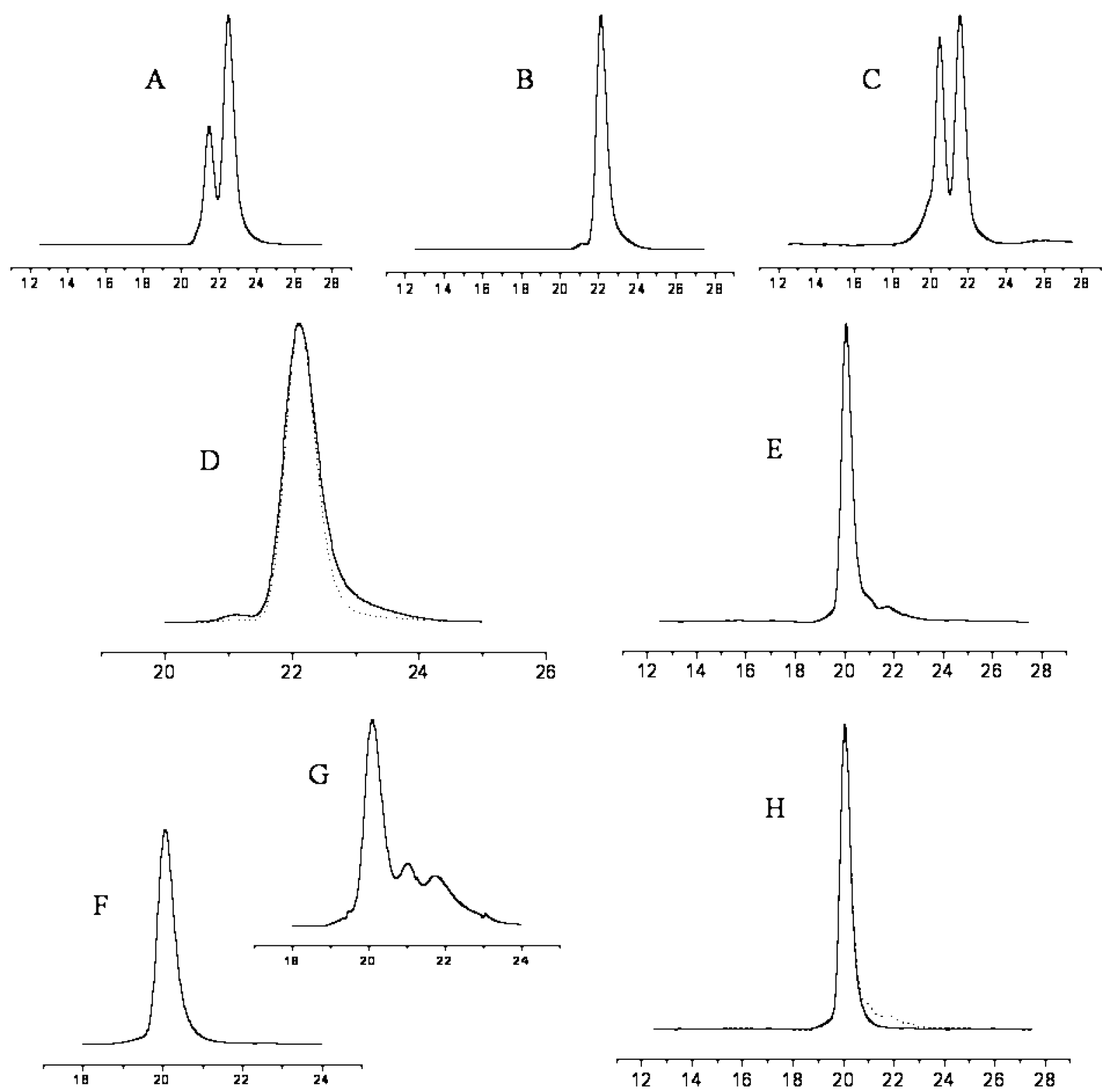

Figure 1. SEC eluograms: (A) PCHDLi +3 equiv of 1,3-PEB in benzene. (B) PCHDLi +1.25 equiv of 1,4-PEB in benzene. (C) First attempt of adding PSLi + PCHD-PEB. (D) Overlay of RI (solid trace) and UV (dotted trace) detectors for the product of PCHDLi $+1,4-\mathrm{PEB}$. (E) Crude product of successful synthesis experiment (PCHD-PEB + colorimetric organolithium titration + PSLi +2 VP). (F) Fractionated final product (down fraction). (G) Material fractionated away from crude product (up-fraction). (H) Overlay of crude (dotted trace, PDI $=1.15)$ and final (solid trace, PDI $=1.04$ ) products. 


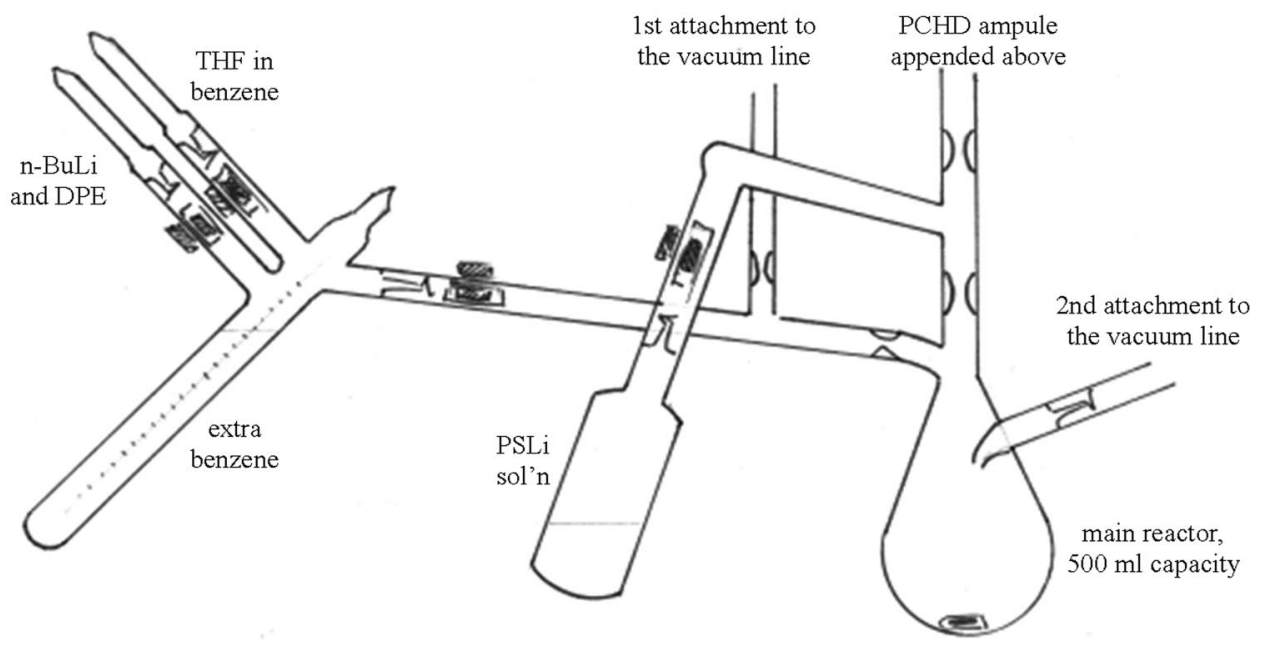

Figure 2. Glass apparatus used for the synthesis of $[\mathrm{PCHD}][\mathrm{PS}][\mathrm{P} 2 \mathrm{VP}]$ miktoarm star terpolymer.

Table 1. Molecular Characteristics of the [PCHD][PS][P2VP] Miktoarm Star Terpolymer ${ }^{a}$

\begin{tabular}{lccccc}
\hline & DR & SEC-LS & SEC-RI & \multicolumn{2}{c}{ calculated values } \\
\cline { 5 - 6 } & $\mathrm{d} n / \mathrm{d} c$ & $M_{\mathrm{w}}, \mathrm{kg} / \mathrm{mol}$ & PDI, $M_{\mathrm{w}} / M_{\mathrm{n}}$ & $M_{\mathrm{w}}, \mathrm{kg} / \mathrm{mol}$ & mass $\%$ \\
\hline PCHD arm & 0.175 & 13.2 & 1.07 & & 24 \\
PS arm & 0.169 & 27.0 & 1.02 & & 49 \\
P2VP arm & & & & 15.1 & 27 \\
$\mu$-star & 0.159 & 55.3 & 1.04 & &
\end{tabular}

${ }^{a} \mathrm{PS}$ is deuterated. MW of P2VP is calculated by $\mathrm{MW}[\mu$-star $]-$ MW[PCHD] - MW[PS].

benzene $\sim 75 / 25$ ). According to our experience, it is desirable to avoid freezing the benzene solution during the in-distillation of THF. The PEB adduct is a diphenyl-stabilized anion and has enough stability toward THF to allow this progression. Then the reactor was stirred until fully chilled to $-78^{\circ} \mathrm{C}$. During the second step 2-vinylpyridine, which was previously purified by distillation from trioctylaluminum, was distilled into the reactor, and polymerization ensued. Finally, the reaction was quenched by the in-distillation of degassed $\mathrm{MeOH}$.

The polymerization to grow the third arm of the miktoarm star terpolymer proceeded without mishap. The crude 3-arm star was $\sim 85 \%$ pure according to SEC profile (see Figure 1E). Fractionation of the crude product by using the solvent system of [toluene-benzene-THF]/hexane as solvent/nonsolvent was rewarded by a final product of higher purity and lower polydispersity. (Again, see the eluogram Figure 1F-H.) The PCHD precursor, the PS precursor, and the finally purified 3-arm star were all characterized by using SEC-RI and SEC light scattering, as shown in Table 1 . These data indicate successful preparation and isolation of a well-defined 3-arm star terpolymer. The compositional analysis was also strengthened somewhat by comparing the results by several ways of measurement, as displayed in Table 2.

Acknowledgment. Research was undertaken fully at Oak Ridge National Laboratory's Center for Nanophase Materials Sciences, which is sponsored by the Scientific User Facilities Division, Office of Basic Energy Sciences, U.S. Department of Energy.

Supporting Information Available: Experimental details. This material is available free of charge via the Internet at http:// pubs.acs.org.
Table 2. Comparative Compositional Analysis of the Final $[$ PCHD $][$ PS $][$ P2VP $] \mu$-Star ${ }^{a}$

\begin{tabular}{lccc}
\hline & $\%$ PCHD & $\%$ PS & $\%$ P2VP \\
\hline by SEC-LS results & 24 & 49 & 27 \\
by exptl gravimetry & 30 & 51 & 19 \\
by ${ }^{1} \mathrm{H}$ and ${ }^{13} \mathrm{C}$ NMR & 26 & 51 & 23
\end{tabular}

${ }^{a}$ PS block is deuterated; for NMR analysis, relative abundance of PCHD to P2VP was established by ${ }^{1} \mathrm{H}$, while abundance of d8PS to P2VP was established by ${ }^{13} \mathrm{C}$.

\section{References and Notes}

(1) Hadjichristidis, N.; Iatrou, H.; Pitsikalis, M.; Pispas, S.; Avgeropoulos, A. Prog. Polym. Sci. 2005, 30, 725-782.

(2) Hadjichristidis, N.; Pitsikalis, M.; Pispas, S.; Iatrou, H. Chem. Rev. 2001, 101, 3747-3792.

(3) Iatrou, H.; Hadjichristidis, N. Macromolecules 1992, 25, 4649-4651.

(4) Bellas, V.; Iatrou, H.; Hadjichristidis, N. Macromolecules 2000, 33, 6993-6997.

(5) Mavroudis, A.; Hadjichristidis, N. Macromolecules 2006, 39, 535540.

(6) Fujimoto, T.; Zhang, H.; Kazama, T.; Isomo, Y.; Hasegawa, H.; Hashimoto, T. Polymer 1992, 33, 2208-2213.

(7) Quirk, R. P.; Kim, Y. J. Polym. Prepr. (Am. Chem. Soc. Polym. Chem. Div.) 1996, 37, 643-644.

(8) Lambert, O.; Reutenauer, S.; Hurtrez, G.; Riess, G.; Dumas, P. Polym. Bull. 1998, 40, 143-149.

(9) Higashihara, T.; Nagura, M.; Inoue, K.; Haraguchi, N.; Hirao, A. Macromolecules 2005, 38, 4577-4587.

(10) Tsoukatos, T.; Hadjichristidis, N. J. Polym. Sci., Part A: Polym. Chem. 2002, 40, 2575-2582.

(11) Wang, X.; Xia, J.; He, J.; Yu, F.; Li, A.; Xu, J.; Lu, H.; Yang, Y. Macromolecules 2006, 39, 6898-6904.

(12) Morton, M.; Fetters, L. J. Rubber Chem. Technol. 1975, 48, 359.

(13) Hadjichristidis, N.; Iatrou, H.; Pispas, S.; Pitsikalis, M. J. Polym. Sci., Part A: Polym. Chem. 2000, 38, 3211.

(14) Hong, K.; Mays, J. W. Macromolecules 2001, 34, 782-786.

(15) Hong, K.; Mays, J. W. Macromolecules 2001, 34, 3540-3547.

(16) Uhrig, D.; Mays, J. W. J. Polym. Sci., Part A: Polym. Chem. 2005, 43, 6179-6222.

(17) Wang, X.; He, J.; Yang, Y. J. Polym. Sci., Part A: Polym. Chem. 2007, 45, 4818-4828.

(18) Paraskeva, S; Hadjichristidis, N J. Polym. Sci., Part A: Polym. Chem. 2000, 38, 931-935.

(19) Poshyachinda, S.; Edwards, H. G. M.; Johnson, A. F. Polymer 1997, $38,535-544$.

MA801852T 